\title{
The PCHIP subdivision scheme
}

\author{
Francesc Aràndiga ${ }^{\mathrm{a}}$, Rosa Donat ${ }^{\mathrm{a}}$, Maria Santágueda $^{\mathrm{b}}$ \\ ${ }^{a}$ Departament de Matemàtica Aplicada. Universitat de València (Spain). \\ ${ }^{b}$ Departament d'Educació. Universitat Jaume I de Castelló de la Plana (Spain).
}

\begin{abstract}
In this paper we propose and analyze a nonlinear subdivision scheme based on the monotononicitypreserving third order Hermite-type interpolatory technique implemented in the PCHIP package in Matlab. We prove the convergence and the stability of the PCHIP nonlinear subdivision process by employing a novel technique based on the study of the generalized Jacobian of the first difference scheme.
\end{abstract}

Keywords: Nonlinear subdivision schemes, convergence, stability, approximation order.

\section{Introduction}

Approximation methods used in scientific and engineering problems are required to represent physical reality as accurately as possible. Good geometric interpolatory reconstructions are most important when the data arise from a physical experiment, since for such data sets geometric considerations, such as preventing spurious behavior near rapid changes in the data, can be even more important than the method's asymptotic accuracy. In many applications, it is often expected that the approximating function reflects the intrinsic shape inferred by the original data set. Nevertheless, good accuracy is still desired to correctly represent smooth behavior.

Subdivision schemes are a powerful tool for the fast generation of curves and surfaces in computeraided geometric design, as well as an essential ingredient in many multiscale algorithms used in data compression, where their potential from the point of view of approximation theory is exploited. Their inherent simplicity has promoted their use also as reconstruction and approximation tools. The convergence and stability properties of these recursive processes are essential for applications, hence it has been, and continues being, a subject of active research.

In many occasions, subdivision schemes are designed (or can be recast) as recursive applications of an approximation technique. In particular, it is well known that the Deslauries-Dubuc [16] (DD henceforth) subdivision schemes fit this general setting, when piecewise polynomial Lagrange interpolation based on a centered stencil is used (see e.g. $[5,9,14,21]$ ). These are canonical examples of data-independent subdivision schemes, which can be described as linear operators between spaces of sequences. The convergence properties of linear interpolatory subdivision schemes is a well understood subject nowadays (see e.g. $[12,17]$ and references therein), as well as their tendency to reconstruct 'discontinuous' discrete data while creating spurious oscillations in the process. In recent years, several nonlinear subdivision schemes have been proposed (see e.g.[2] and references therein) in an attempt to avoid the Gibb's-like oscillatory behavior that occurs when data-independent interpolatory techniques are used for the successive refinement of discrete data that display a nearly discontinuous behavior. On the other hand, the need to obtain subdivision processes that preserve the shape of the original discrete data has been considered in [23? ], from a different perspective.

It is well known that the analysis of nonlinear subdivision schemes cannot be carried out with the same techniques as in the linear case (see e.g. [18] and references therein). In some cases, a successful

Email addresses: arandiga@uv.es (Francesc Aràndiga), donat@uv.es (Rosa Donat), santague@uji.es (Maria Santágueda)

Preprint submitted to Applied Mathematics and Computation

June 9, 2015 
way to study the convergence properties of a nonlinear subdivision scheme follows from the ability to write the nonlinear scheme as a perturbation of a standard linear subdivision scheme whose convergence properties are known. It is shown in [3] that, in such cases, convergence and/or stability can be obtained if the perturbation satisfies certain properties (see Theorem 1. On the other hand, a novel approach, based on the analysis of the properties difference schemes, has been recently proposed in [? ] in order to analyze the stability properties on certain nonlinear subdivision schemes.

In this paper we define and analyze a nonlinear scheme based on the monotononicity-preserving third order Hermite-type interpolatory technique implemented in the Matlab PCHIP function [6]. We prove that, as expected, the resulting subdivision scheme, named also PCHIP, is monotonicity preserving and can be written as a nonlinear perturbation of a linear scheme. Since it can easily be shown to admit a first difference scheme, we analyze its convergence and stability by using the theory developed in [27], where the approach outlined in [20] is developed to study convergence and stability of subdivision schemes that can be written as a certain nonlinear perturbation of a linear, convergent, subdivision scheme.

The paper is organized as follows: in section 2 we briefly recall the standard framework for the construction of interpolatory subdivision schemes based on piecewise polynomial interpolatory techniques. Section 3 recalls well known results about monotonicity preserving Hermite-type interpolation in general, and the PCHIP interpolatory technique in particular. In section 4 we describe the PCHIP subdivision scheme and study its convergence and stability, as well as its approximation order for smooth data. Finally, in section 5 we present several numerical examples that illustrate our theoretical results, while showing that the PCHIP subdivision scheme behaves in a non-oscillatory manner when refining discrete discontinuous data. We close in section 6 with some conclusions and future perspectives.

\section{Interpolatory Subdivision based on piecewise polynomial reconstructions}

A general setting by which a piecewise polynomial interpolatory technique can be used to provide the set of local rules that defines an interpolatory subdivision scheme has been described in [5, 14, 21]. For the sake of completeness, we briefly review the main steps of this general setting: Assume that $\chi^{l} \subset \chi^{l+1}$ are two nested grids on $\mathbb{R}$. If $\left\{f^{l}\right\}$ is a set of known data associated to the grid $\chi^{l}$, and $\mathcal{I}[x, \cdot]$ is a piecewise polynomial reconstruction technique, new data associated to the grid $\chi^{l+1}$ can be generated as follows

$$
f_{i}^{l+1}=\mathcal{I}\left[x_{i}^{l+1}, f^{l}\right], \quad \text { for } x_{i}^{l+1} \in \chi^{l+1} .
$$

This process defines a recursive subdivision scheme where sequences of values on denser and denser meshes are obtained, according to the set of local rules derived from $\mathcal{I}[x, \cdot]$.

Let us assume that the refinement factor between the (1-d) nested grids is 2 (i.e. $x_{i}^{l+1}=x_{2 i}^{l}$ ). If $\mathcal{I}\left[x, f^{l}\right]$ interpolates the values $\left\{f^{l}\right\}$ on the grid $\chi^{l}$ we have

$$
f_{i}^{l+1}=\mathcal{I}\left[x_{i}^{l+1}, f^{l}\right]=\mathcal{I}\left[x_{2 i}^{l}, f^{l}\right]=f_{2 i}^{l} \quad \text { for each } x_{i}^{l+1}=x_{2 i}^{l} \in \chi^{l},
$$

hence the values on a given mesh are 'copied' at the same location on higher resolution levels, while

$$
f_{i}^{l+1}=\mathcal{I}\left[x_{i}^{l+1}, f^{l}\right] \quad \text { for } x_{i}^{l+1} \in \chi^{l+1} \backslash \chi^{l}
$$

specifies the local rules used for the generation of new data values.

These subdivision schemes are linear operators between spaces of bounded sequences and their convergence properties are well known, as it is well known that, for data-independent interpolatory techniques) they do not preserve the shape of the original data to be refined, when the degree of the polynomial pieces is larger than 1. For this reason, nonlinear piecewise polynomial interpolatory techniques have been considered in the literature, within this same framework, in an attempt to construct interpolatory subdivision schemes that avoid the Gibbs-like behavior displayed by DD schemes when applied to discrete data with large gradients.

Examples of non-linear, non-oscillatory subdivision schemes that fit this general framework are the ENO-WENO subdivision schemes described in $[8,7,13]$ or the Piecewise Parabolic Hyperbolic $(\mathrm{PPH})$ 
scheme in [2]. The Power schemes $[3,14,25]$ and the nonlinear monotonicity preserving schemes developed in [24], which do not fit within this framework, are also examples of non-oscillatory subdivision schemes. For all these schemes, the study of basic questions related to convergence and stability require specific tools that are different from those traditionally used in the analysis of linear schemes.

It is well known that the classical notion of convergence, i.e. the existence of a continuous function which is the limit of the polygonal functions that interpolate the $k$-level data $S^{k} f$, for any initial data $f$, is equivalent to the Lipschitz stability of the scheme in the linear case, but this not so for nonlinear subdivision schemes (see e.g. [24, 18] and references therein). The Power $_{p}$ schemes $[2,4,14]$, as well as other related subdivision schemes considered in $[1,15]$, can be written in the following general form

$$
\left(S_{\mathcal{N}} f\right)_{n}=\left(S_{\mathcal{L}} f\right)_{n}+\mathcal{F}(\delta f)_{n}, \quad f \in l_{\infty}(\mathbb{Z}), \quad n \in \mathbb{Z}
$$

where $\mathcal{F}: l^{\infty}(\mathbb{Z}) \rightarrow l^{\infty}(\mathbb{Z})$ may be a nonlinear operator, $\delta: l^{\infty}(\mathbb{Z}) \rightarrow l^{\infty}(\mathbb{Z})$ is linear and continuous and $S_{\mathcal{L}}$ is a linear and convergent subdivision scheme. In $[2,4,1,15]$, the convergence and stability of the proposed schemes are systematically analyzed by using the following results.

Theorem 1. Let $S_{\mathcal{N}}$ be a nonlinear subdivision scheme which can be written in the form (3).

The scheme $S_{\mathcal{N}}$ is uniformly convergent provided that $\mathcal{F}$, and $\delta$ satisfy the following conditions:

$$
\begin{array}{lllr}
\text { C1. } & \exists M>0: & \|\mathcal{F}(f)\|_{\infty} \leq M\|f\|_{\infty} & \forall f \in l^{\infty}(\mathbb{Z}) \\
\text { C2. } & \exists L>0,0<T<1: & \left\|\delta S_{\mathcal{N}}^{L}(f)\right\|_{\infty} \leq T\|\delta f\|_{\infty} & \forall f \in l^{\infty}(\mathbb{Z})
\end{array}
$$

Moreover, if $S_{\mathcal{L}}$ is $C^{\alpha-}$ convergent ${ }^{1}$ then $S_{\mathcal{N}}$ is at least $C^{\beta-}$ convergent with $\beta=\min \left\{-\frac{\log _{2}(T)}{L}, \alpha\right\}$.

The scheme $S_{\mathcal{N}}$ is stable provided that $\mathcal{F}$ and $\delta$ satisfy,

$$
\begin{array}{lll}
\text { S1. } & \exists M>0: & \|\mathcal{F}(f)-\mathcal{F}(g)\|_{\infty} \leq M\|f-g\|_{\infty} \quad \forall f, g \in l^{\infty}(\mathbb{Z}) \\
\text { S2. } & \exists L>0,0<T<1: & \left\|\delta\left(S_{\mathcal{N}}^{L}(f)-S_{\mathcal{N}}^{L}(g)\right)\right\|_{\infty} \leq T\|\delta(f-g)\|_{\infty}, \forall f, g \in l^{\infty}(\mathbb{Z})
\end{array}
$$

For nonlinear schemes, stability is important also in order to determine the approximation order of the subdivision scheme.

Remark 1. $S$ is said to have approximation order $p$ after one iteration if, for any $f(x)$ sufficiently smooth, it satisfies

$$
\max _{i}\left|(S f)_{i}-f(i h / 2)\right| \leq C h^{q}, \quad C<\infty, \quad f^{0}=\{f(j h)\}_{j \in \mathbb{Z}}
$$

The order of approximation after one iteration of the interpolatory subdivision schemes described by (2) is given by the error formula of the interpolatory technique $\mathcal{I}[x, \cdot]$.

Theorem 2. [23] Let $S$ be a convergent and stable subdivision scheme.

If $S$ reproduces polynomials of degree $q-1 \geq 0$. Then, $S$ has approximation order $q$, i.e.

$$
\left\|S^{\infty} f-f\right\|_{\infty} \leq D h^{q} .
$$

If $S$ satisfies (4), then $S$ has approximation order $q$.

\section{Monotonicity-preserving Hermite Interpolation}

A typical demand is that of producing a monotone function while fitting monotone data, and several monotone interpolatory techniques have been proposed in the literature that involve the construction of monotone piecewise polynomial interpolatory functions (see e.g. $[10,19,22]$ ). Accuracy and monotonicity

\footnotetext{
${ }^{1}$ For $0<\alpha \leq 1, C^{\alpha-}$ is the space of bounded continuous functions satisfying $|f(x)-f(y)| \leq C|x-y|^{\alpha_{1}}, \forall \alpha_{1}<\alpha$, $\forall x, y \in \mathbb{R}$, with $C>0$ independent of $x, y$. For $\alpha>1, \alpha=p+r, p \in \mathbb{N}, 0<r \leq 1$, it is required that $f^{(p)} \in C^{r-}$
} 
preservation are somewhat conflicting requirements. We review below some known results concerning Monotone Hermite Interpolation and refer the interested reader to [10, 19, 22], and references therein for further information on this subject.

The classical problem of constructing a cubic polynomial, $P_{i}(x)$, that interpolates function values and derivative values at two nodes, $x_{i}, x_{i+1}$

$$
P_{i}\left(x_{i}\right)=f_{i}, \quad P_{i}\left(x_{i+1}\right)=f_{i+1}, \quad P_{i}^{\prime}\left(x_{i}\right)=\dot{f}_{i}, \quad P_{i}^{\prime}\left(x_{i+1}\right)=\dot{f}_{i+1}
$$

can be uniquely determined by using, for example, the Newton form of $P_{i}(x)$,

$$
P_{i}(x)=c_{1}+c_{2}\left(x-x_{i}\right)+c_{3}\left(x-x_{i}\right)^{2}+c_{4}\left(x-x_{i}\right)^{2}\left(x-x_{i+1}\right) .
$$

The interpolatory conditions (6) easily lead to the following set of coefficients

$$
c_{1}=f_{i}, \quad c_{2}=\dot{f}_{i}, \quad c_{3}=\left(m_{i}-\dot{f}_{i}\right) / h_{i}, \quad c_{4}=\left(\dot{f}_{i+1}+\dot{f}_{i}-2 m_{i}\right) / h_{i}^{2}
$$

with $m_{i}:=\nabla f_{i} / h_{i}, \nabla f_{i}=\left(f_{i+1}-f_{i}\right), h_{i}=x_{i+1}-x_{i}$. Notice that

$$
\operatorname{sgn}\left(\dot{f}_{i}\right)=\operatorname{sgn}\left(\dot{f}_{i+1}\right)=\operatorname{sgn}\left(\nabla f_{i}\right)
$$

is a necessary (but obviously not sufficient) condition for the monotonicity of $P_{i}(x)$ satisfying (6). To ensure monotonicity, additional constraints on the $\left\{\dot{f}_{i}\right\}$ values are required, [10, 19, 22]. Among these constraints, the simplest one (and probably the most used) is the de-Boor and Swarz condition [10],

$$
0 \leq \alpha_{i}, \beta_{i} \leq 3, \quad \alpha_{i}=\frac{\dot{f}_{i}}{m_{i}}, \beta_{i}=\frac{\dot{f}_{i+1}}{m_{i}}
$$

which ensures that (7)-(8) is monotone. Hence, the key to monotonicity relies on the definition of appropriately constrained derivative values, which should be as accurate as possible.

The interested reader is referred to [6] for various alternatives to construct limited derivative values. In this paper we shall consider Brodlie's formula [19] for the approximation of derivative values. For equally spaced nodes

$$
\dot{f}_{i}^{B}=\mathrm{H}\left(m_{i-1}, m_{i}\right), \quad \mathrm{H}(x, y)=\left\{\begin{array}{ll}
\frac{2 x y}{x+y} & x y>0 \\
0 & x y \leq 0
\end{array} .\right.
$$

The following properties of $H(x, y)$ are easily shown

$$
\text { (a) }|H(x, y)| \leq \max \{|x|,|y|\}, \quad \text { (b) }|H(x, y)| \leq 2 \min \{|x|,|y|\}
$$

Notice that (12)-(b)implies that $\left|\dot{f}_{i}^{B}\right| \leq 2 \min \left(\left|m_{i-1}\right|,\left|m_{i}\right|\right)$, hence (10) is satisfied and $P_{i}(x)$ is monotonicity preserving. This Hermite interpolant is implemented in Matlab's PCHIP function [26].

\subsection{The Harmonic Mean: Generalized Gradients}

It is easy to see that $\mathrm{H}(x, y)$ in $(11)$ is a continuous function that is differentiable almost everywhere in $\mathbb{R}^{2}$. For $x y>0$ we have

$$
\nabla \mathrm{H}(x, y)=\frac{2}{(x+y)^{2}}\left(y^{2}, x^{2}\right)=(\phi(t), \phi(-t)), \quad t:=\frac{y-x}{y+x}, \quad \phi(t):=\frac{1}{2}(1+t)^{2},
$$

(notice that $x y>0 \leftrightarrow t \in[-1,1]$ ). Moreover $\nabla \mathrm{H}(x, y)$ in $(13)$ satisfies

$$
\lim _{y \rightarrow 0} \partial_{x} \mathrm{H}(x, y)=0 \quad \forall x \neq 0 \quad \lim _{x \rightarrow 0} \partial_{y} \mathrm{H}(x, y)=0 \quad \forall y \neq 0 .
$$

As observed in [20], these properties imply that $\mathrm{H}(x, y)$ in $(11)$ belongs to the class of $C_{p w}^{1}\left(\mathbb{R}^{2}\right)$-functions (see $[20,27])$. Functions, $\psi: \mathbb{R}^{2} \rightarrow \mathbb{R}$, in this class are continuous, piecewise smooth and have directional 
derivatives except (maybe) at $(0,0)$ and across certain hyperplanes (straight lines in $\mathbb{R}^{2}$ ), that separate regions of $C^{1}$ smoothness. On the other hand, directional derivatives along the separating hyperplanes do exist. For $H(x, y)$, the hyperplanes separating the regions of $C^{1}$ smoothness in $\mathbb{R}^{2}$ are the coordinate axis and it is easy to check that $D_{(1,0)}(x, 0)=0=D_{(0,1)}(0, y)$, for $x \neq 0 \neq y[27]$.

A function $\psi \in C_{p w}^{1}\left(\mathbb{R}^{2}\right)$ admits a Generalized Gradient, $D \psi(x, y)$, at each $(x, y) \in \mathbb{R}^{2}$. As the 'classical' gradient, $D \psi(x, y)$ is characterized by the fact that the associated linear map recovers all directional derivatives that 'make sense'. More concretely for $0 \neq v \in \mathbb{R}^{2},(0,0) \neq(x, y) \in \mathbb{R}^{2}$

$$
D_{v} \psi(x, y)=D \psi(x, y)
$$

for any vector $v \neq 0$ when $(x, y)$ is in a smoothness region, and for any $v \in \mathcal{H}$, a hyperplane separating two smoothness regions, when $(x, y) \in \mathcal{H}$. The interested reader is referred to [27] for a detailed analysis on these facts and their consequences. As in the case of the classical gradient of a $C^{1}$ function, (15) leads to the following fundamental result (see [27] for the proof).

Theorem 3. Let $\gamma:[a, b] \rightarrow \mathbb{R}^{2}$ be a continuous curve, differentiable a.e. in $(a, b), \psi: \mathbb{R}^{2} \rightarrow \mathbb{R} a$ function in $C_{p w}^{1}\left(\mathbb{R}^{2}\right)$ and $D \psi$ a generalized gradient of $\psi$. Then $\tilde{\gamma}=\psi \circ \gamma:[a, b] \rightarrow \mathbb{R}$ is also a continuous curve, differentiable a.e. in $(a, b)$ and

$$
\tilde{\gamma}^{\prime}(t)=D \psi(\gamma(t)) \gamma^{\prime}(t) \quad \text { a.e. in }[a, b]
$$

For $\mathrm{H}(x, y)$ in $(11)$, the compatibility conditions (14) guarantee that all the directional derivatives that exist at any point $(0,0) \neq(x, y) \in \mathbb{R}^{2}$ can be recovered, in the usual sense, from the following generalized gradient, defined, at each point $(x, y) \in \mathbb{R}^{2}$, as follows (see $[27,20]$ )

$$
D \mathrm{H}(x, y)=\left(D_{x} \mathrm{H}, D_{y} \mathrm{H}\right)(x, y)= \begin{cases}(0,0) & \text { if } x y \leq 0 \\ \nabla \mathrm{H}(x, y) & \text { if } x y>0 .\end{cases}
$$

The following corollary is a well known fact which follows easily from the previous theorem and the fact that $\nabla \mathrm{H}(x, y)$ in (13) is uniformly bounded.

Corollary 1. The harmonic mean satisfies

$$
\left\|H\left(x_{1}, y_{1}\right)-H\left(x_{2}, y_{2}\right)\right\|_{\infty} \leq 2\left\|\left(x_{1}, y_{1}\right)-\left(x_{2}, y_{2}\right)\right\|_{\infty} \quad \forall\left(x_{i}, y_{i}\right) \in \mathbb{R}^{2} .
$$

Proof. Consider $\gamma:[0,1] \rightarrow \mathbb{R}, \quad \gamma(t):=t\left(x_{1}, y_{1}\right)+(1-t)\left(x_{2}, y_{2}\right)$. Theorem 3 allows us to write

$$
H\left(x_{1}, y_{1}\right)-H\left(x_{2}, y_{2}\right)=\gamma(1)-\gamma(0)=\int_{0}^{1} D \mathrm{H}(\gamma(t)) \gamma^{\prime}(t) d t
$$

Taking into account (17) and (13), since $0 \leq \phi( \pm t) \leq 2, \forall t \in[-1,1]$ (see Figure 1) we get

$$
0 \leq D_{x} \mathrm{H}(x, y) \leq 2, \quad 0 \leq D_{y} \mathrm{H}(x, y) \leq 2, \quad\|D \mathrm{H}(x, y)\|_{1} \leq 2, \quad \forall(x, y) \in \mathbb{R}^{2} .
$$

Since $\gamma^{\prime}(t)=\left(x_{1}, y_{1}\right)-\left(x_{2}, y_{2}\right)$, the result follows from the bounds above.

The schemes considered in this paper are binary schemes of the form

$$
(S f)_{2 j+k}=\psi_{k}\left(f_{j-p}, \ldots, f_{j+p}\right), k=0,1 \quad f \in l_{\infty}(\mathbb{Z}), j \in \mathbb{Z},
$$

where the functions $\psi_{k}: \mathbb{R}^{2 p+1} \rightarrow \mathbb{R}$ can be expressed as linear combinations of functions that are either differentiable in $\mathbb{R}^{2 p+1}$, or of the form $\mathrm{H} \circ M$ with $M \in \mathbb{R}^{2 \times(2 p+1)}$ a linear map of full rank. It is easy to shown that these functions also admit a generalized gradient that satisfies (16). 
Corollary 2. Let $\psi \in C_{p w}^{1}\left(\mathbb{R}^{2}\right), M \in \mathbb{R}^{2 \times p}$ such that $\operatorname{rank}(M)=2 \leq p$, and $\tilde{\psi}:=\psi \circ M: \mathbb{R}^{p} \rightarrow \mathbb{R}$. Then $D \tilde{\psi}(y):=D \psi(M(y)) M, \forall y \in \mathbb{R}^{p}$, satisfies

$$
(\tilde{\psi} \circ \gamma)^{\prime}(t)=D \tilde{\psi}(\gamma(t)) \gamma^{\prime}(t) \quad \text { a.e. in }(a, b)
$$

for any $\gamma:[a, b] \rightarrow \mathbb{R}^{p}$ Lipschitz.

Proof. To prove (20), we apply Theorem 3 to $\psi \in C_{p w}^{1}\left(\mathbb{R}^{2}\right)$ and $\gamma_{M}:=M \circ \gamma:[a, b] \rightarrow \mathbb{R}^{2}$, which is a Lipschitz curve satisfying $\gamma_{M}^{\prime}(t)=M \gamma^{\prime}(t)$ a.e. in $(a, b)$. Then,

$$
(\tilde{\psi} \circ \gamma)^{\prime}(t)=\left(\psi \circ \gamma_{M}\right)(t)=D \psi\left(\gamma_{M}(t)\right) \gamma_{M}^{\prime}(t)=D \tilde{\psi}(\gamma(t)) \gamma^{\prime}(t) \quad \text { a.e. in }(a, b)
$$

Remark 2. In the above corollary, if $\|D \psi(x)\|_{1} \leq C, \forall x \in \mathbb{R}^{2}$ then

$$
\|D \tilde{\psi}(y)\|_{1}=\|D \psi(M y) M\|_{1} \leq\left\|M^{T}\right\|_{1}\|D \psi(M y)\|_{1}, \quad \forall y \in \mathbb{R}^{p}
$$

hence $\|D \tilde{\psi}(y)\|_{1}$ is also uniformly bounded.

The class of functions $\tilde{\psi}: \mathbb{R}^{n} \rightarrow \mathbb{R}$ that can be written as a linear combination of functions of the form $\psi \circ M$, with $\psi \in C_{p w}^{1}\left(\mathbb{R}^{2}\right)$ and $M: \mathbb{R}^{n} \rightarrow \mathbb{R}^{2}$ a full rank matrix is referred to as $C_{p w}^{1}\left(\mathbb{R}^{n}\right)$ in [27].

\section{PCHIP subdivision}

Let us consider the subdivision scheme associated to the piecewise polynomial interpolatory technique obtained from the cubic Hermite polynomials (6), with derivative values computed by Brodlie's formula (11). A straightforward computation leads to

$$
P_{i}\left(x_{i+1 / 2}\right)=f_{i}+\frac{1}{2} \nabla f_{i}+\frac{1}{8}\left(\mathrm{H}\left(\nabla f_{i-1}, \nabla f_{i}\right)-\mathrm{H}\left(\nabla f_{i}, \nabla f_{i+1}\right)\right)
$$

hence, the resulting interpolatory subdivision scheme (associated to the Matlab PCHIP function, hence the name) can be written as follows

$$
\left\{\begin{array}{l}
(\mathrm{S} f)_{2 n}=f_{n} \\
(\mathrm{~S} f)_{2 n+1}=\frac{f_{n}+f_{n+1}}{2}+\frac{1}{8}\left(\mathrm{H}\left(\nabla f_{n-1}, \nabla f_{n}\right)-\mathrm{H}\left(\nabla f_{n}, \nabla f_{n+1}\right)\right) .
\end{array}\right.
$$

We readily notice that this scheme has the general form (3), with $S_{\mathcal{L}}$ the linear scheme obtained from piecewise polynomial interpolation of degree $1, \delta=\nabla$ and

$$
\mathcal{F}(f)_{2 n}=0, \quad \mathcal{F}(f)_{2 n+1}=\frac{1}{8}\left(\mathrm{H}\left(f_{n-1}, f_{n}\right)-\mathrm{H}\left(f_{n}, f_{n+1}\right)\right) .
$$

Since the scheme has been defined from a monotonicity preserving interpolatory technique, we expect that it inherits this property. It is easy to check that this scheme is monotonicity preserving making use of the first difference scheme, $S^{[1]}$, which is completely characterized by the condition $\nabla S=S^{[1]} \nabla$. The first difference scheme for the PCHIP subdivision process can easily be deduced:

$$
\left\{\begin{aligned}
\left.\mathrm{S}^{[1]} w\right)_{2 n} & =\frac{w_{n}}{2}+\frac{1}{8}\left(\mathrm{H}\left(w_{n-1}, w_{n}\right)-\mathrm{H}\left(w_{n}, w_{n+1}\right)\right) \\
\left(\mathrm{S}^{[1]} w\right)_{2 n+1} & =\frac{w_{n}}{2}-\frac{1}{8}\left(\mathrm{H}\left(w_{n-1}, w_{n}\right)-\mathrm{H}\left(w_{n}, w_{n+1}\right)\right)
\end{aligned} \quad \forall n \in \mathbf{Z}\right.
$$


Notice that $\forall f \in l_{\infty}(\mathbb{Z}), \mathrm{S}^{[1]} f$ in $(24)$ can be written as follows

$$
\left(\mathrm{S}^{[1]} f\right)_{2 n+k}=\psi_{k}\left(f_{n-1}, f_{n}, f_{n+1}\right) \quad j \in \mathbb{Z}, \quad \psi_{k}(x, y, z)=\frac{y}{2}+(-1)^{k} \frac{1}{8}\left(\mathrm{H} \circ M_{1}-\mathrm{H} \circ M_{2}\right)(x, y, z)
$$

where $M_{1}(x, y, z)=(x, y), M_{2}(x, y, z)=(y, z)$. Hence, the functions $\psi_{k}$ can be expressed as linear combinations of functions that are either differentiable in $\mathbb{R}^{3}$ (with uniformly bounded gradients), or of the form $H \circ M$ with $M \in \mathbb{R}^{2 \times 3}$ a linear map of full rank. We can compute (uniformly bounded) generalized gradients of these functions by the chain rule, using Corollary 2. Since $\left.\psi_{0}(x, y, z)+\psi_{1}(x, y, z)\right)=y$, we easily deduce that

$$
\begin{aligned}
D \psi_{0}(x, y, z) & =\frac{1}{8}\left(D_{x} \mathrm{H}(x, y), 4+D_{y} \mathrm{H}(x, y)-\mathrm{H}(y, z),-D_{y} \mathrm{H}(y, z)\right) \\
D \psi_{1}(x, y, z) & =(0,1,0)-D \psi_{0}(x, y, z)
\end{aligned}
$$

Lemma 4. The PCHIP scheme is monotonicity preserving.

Proof. Let $f \in l_{\infty}(\mathbb{Z}): \nabla f_{i}>0, \forall i$. Since $\nabla S f=S^{[1]} \nabla f, S$ is monotonicity preserving if and only if $S^{[1]}$ is positivity preserving, i.e. it keeps positive data positive. Let us assume $w_{n} \geq 0, \forall n \in \mathbb{Z}$. Taking into account that $\mathrm{H}(x, y) \geq 0$ for $x, y \geq 0$ and (12)-(b), we can write

$$
\left(\mathrm{S}^{[1]} w\right)_{2 n}=\frac{w_{n}}{2}+\frac{1}{8}\left(\mathrm{H}\left(w_{n-1}, w_{n}\right)-\mathrm{H}\left(w_{n}, w_{n+1}\right)\right) \geq \frac{w_{n}}{2}-\frac{1}{8} \mathrm{H}\left(w_{n}, w_{n+1}\right) \geq \frac{w_{n}}{2}-\frac{2}{8} w_{n} \geq 0 .
$$

We prove that $\left(\mathrm{S}^{[1]} w\right)_{2 n+1} \geq 0$ in a completely analogous manner.

Remark 3. It is straightforward to see that the PCHIP scheme reproduces polynomials of degree 1 exactly. However, as the scheme is non-linear, this property does not guarantee the existence of the second difference scheme $S^{[2]}[20]$.

\subsection{Convergence}

Theorem 5. The PCHIP subdivision scheme (22) is convergent.

Proof. We check condition $\mathbf{C 1}$ in Theorem 1 for $\mathcal{F}$ in (23). Using (12)-(a) we get

$$
\left.\left|(\mathcal{F}(f))_{2 n+1}\right| \leq \frac{1}{8}\left(\max \left\{\left|f_{n-1}\right|,\left|f_{n}\right|\right\}+\max \left\{\left|f_{n}\right|,\left|f_{n+1}\right|\right\}\right) \leq \frac{1}{4}\|f\|_{\infty}, \quad \forall f \in l_{\infty}(\mathbb{Z}), \forall n \in \mathbb{Z}\right)
$$

We rewrite condition $\mathbf{C 2}$ in Theorem 1 in terms of $S^{[1]}$. Taking into account that

$$
\delta S^{L}=\nabla S S^{L-1}=S^{[1]} \nabla S^{L-1}=\left(S^{[1]}\right)^{L} \nabla,
$$

C2 is equivalent to the following condition

$$
\exists L>0,0<T<1: \quad\left\|\left(S^{[1]}\right)^{L}(f)\right\|_{\infty} \leq T\|f\|_{\infty} \quad \forall f \in l^{\infty}(\mathbb{Z})
$$

We check now that (29) holds for $L=1$. Notice that

$$
\begin{gathered}
\left(\mathrm{S}^{[1]} w\right)_{2 n}=Z_{+}\left(w_{n-1}, w_{n}, w_{n+1}\right), \quad\left(\mathrm{S}^{[1]} w\right)_{2 n+1}=Z_{-}\left(w_{n-1}, w_{n}, w_{n+1}\right), \\
Z_{ \pm}\left(x_{1}, x_{2}, x_{3}\right)=\frac{x_{2}}{2} \pm \frac{1}{8}\left(\mathrm{H}\left(x_{1}, x_{2}\right)-\mathrm{H}\left(x_{2}, x_{3}\right)\right) .
\end{gathered}
$$

Using that $|\mathrm{H}(x, y)| \leq \max \{|x|,|y|\}$, it is easy to see that $\forall x=\left(x_{1}, x_{2}, x_{3}\right) \in \mathbb{R}^{3}$,

$$
\left|Z_{ \pm}(x)\right| \leq \frac{5}{8}\|x\|_{\infty}, \quad \Rightarrow \quad\left\|\mathrm{S}^{[1]} w\right\|_{\infty} \leq \frac{5}{8}\|w\|_{\infty}
$$


Hence, by Theorem 1, we obtain the result.

To prove (31), we notice that $Z_{ \pm}\left(x_{1}, x_{2}, x_{3}\right)=-Z_{ \pm}\left(-x_{1},-x_{2},-x_{3}\right)$, hence is sufficient to prove it for $x_{2}>0$. In this case, $\mathrm{H}\left(x_{1}, x_{2}\right) \geq 0, \mathrm{H}\left(x_{2}, x_{3}\right) \geq 0$ and we can write

$$
-\frac{1}{8}\|x\|_{\infty} \leq-\frac{1}{8} \mathrm{H}\left(x_{2}, x_{3}\right) \leq Z_{+}\left(x_{1}, x_{2}, x_{3}\right) \leq \frac{x_{2}}{2}+\frac{1}{8} \mathrm{H}\left(x_{1}, x_{2}\right) \leq \frac{5}{8}\|x\|_{\infty}
$$

i.e. (31) holds for $Z_{+}$. The proof for $Z_{-}$is absolutely similar.

Remark 4. Notice that Theorem 1 implies that $S^{\infty} f \in C^{\alpha-}(\mathbb{R})$ with $\alpha \geq 0.678$

\subsection{Stability: Generalized Gradients and Generalized Jacobians}

It is seen in $[20,27]$ that any scheme $S$ that is defined by functions that admit uniformly bounded generalized gradients admits a Generalized Jacobian, i.e. a bounded linear map $D S: l_{\infty}(\mathbb{Z}) \rightarrow \mathcal{L}\left(l_{\infty}(\mathbb{Z})\right)$, where $\mathcal{L}\left(l_{\infty}(\mathbb{Z})\right)$ is the space of bounded linear operators $\mathcal{M}: l_{\infty}(\mathbb{Z}) \rightarrow l_{\infty}(\mathbb{Z})$. For a binary scheme (19) $D S(f)$ is the linear operator associated to the bi-infinite matrix whose rows have the following non-zero components

$$
(D S(f))_{[2 j+k, j-p: j+p]}=D \psi_{k}\left(f_{j-p}, \ldots, f_{j+p}\right), \quad k=0,1, \quad j \in \mathbb{Z},
$$

where $D \psi_{k}$ is the a generalized gradient of the function $\psi_{k}$. Obviously, if the generalized gradients of the functions involved are uniformly bounded, then $\|D S(f)\|_{\infty} \leq C$, with $C$ independent of $f \in l_{\infty}(\mathbb{Z})$. We state also the following fundamental result (see [27] for a detailed proof).

Theorem 6. Let $S$ be a scheme defined by local rules than can be expressed as $C_{p w}^{1}\left(\mathbb{R}^{n}\right)$ functions, for some $n$, with uniformly bounded gradients. Let $\gamma:[a, b] \rightarrow l_{\infty}(\mathbb{Z})$ be Lipschitz Curve ${ }^{2}$. Then $\tilde{\gamma}=S \circ \gamma$ : $[a, b] \rightarrow l_{\infty}(\mathbb{Z})$ is also a Lipschitz curve, and $\tilde{\gamma}^{\prime}(t)=D S(\gamma(t)) \gamma^{\prime}(t)$ a.e. on $(a, b)$.

This theorem allows to study the contractivity properties of the powers of certain subdivision schemes, by the following argument, sketched in $[20]$. Given $f, g \in l_{\infty}(\mathbb{Z})$, define recursively $\gamma^{j}:[0,1] \rightarrow l_{\infty}(\mathbb{Z})$ as follows

$$
\gamma^{0}(t)=t f+(1-t) g \quad \gamma^{j}(t)=S \circ \gamma^{j-1}(t) \quad j>0 .
$$

Notice that $\gamma^{0}(1)=f, \gamma^{0}(0)=g, \gamma^{j}(1)=S^{j} f, \gamma^{j}(0)=S^{j} g$. Notice that $\gamma^{0}(t)$ is Lipschitz. Under the conditions specified in Theorem $6, \gamma^{j}$ is Lipschitz and

$$
\begin{aligned}
\left(\gamma^{0}\right)^{\prime}(t) & =f-g \\
\left(\gamma^{j}\right)^{\prime}(t) & =D S\left(\gamma^{j-1}(t)\right)\left(\gamma^{j-1}(t)\right)^{\prime}=\ldots \\
& =D S\left(\gamma^{j-1}(t)\right) D S\left(\gamma^{j-2}\right)(t) \cdots D S\left(\gamma^{0}(t)\right)\left(\gamma^{0}\right)^{\prime}(t), \quad \text { a.e. } \operatorname{in}(0,1)
\end{aligned}
$$

Hence, we can write

$$
\begin{aligned}
S^{j} f-S^{j} g=\gamma^{j}(1)-\gamma^{j}(0) & =\int_{0}^{1} D S\left(\gamma^{j-1}(t)\right) D S\left(\gamma^{j-2}\right)(t) \cdots D S\left(\gamma^{0}(t)\right)(f-g) d t, \\
\left\|S^{j} f-S^{j} g\right\|_{\infty} & \leq\left(\int_{0}^{1}\left\|\Pi_{k=0}^{j-1} D S\left(\gamma^{k}(t)\right)\right\|_{\infty} d t\right)\|f-g\|_{\infty} .
\end{aligned}
$$

According to (34), the contractivity of $S^{j}$ can be determined by studying the bounds on the successive products of Generalized Jacobians of $S$. This strategy has been successfully used to study the stability of the Power $_{p}$ nonlinear schemes in [20,27], and shall be used next in order to show the stability of the PCHIP subdivision scheme.

\footnotetext{
${ }^{2} \gamma=\left\{\gamma_{i}\right\}_{i \in \mathbb{Z}}$ with $\gamma_{i}: \mathbb{R} \rightarrow \mathbb{R}$ and $\left|\gamma_{i}(x)-\gamma_{i}(y)\right| \leq M|x-y|, \forall x, y \in \mathbb{R}, \forall i \in \mathbb{Z}$
} 
Theorem 7. The PCHIP subdivision scheme (22) is stable.

Proof. Condition S1 is obviously satisfied for $\mathcal{F}$ in $(23)$, since $\mathrm{H}(x, y)$ is a Lipschitz function. Notice that we can rewrite $\mathbf{S 2}$ in Theorem 1 in terms of $\mathrm{S}^{[1]}$, using (28). The equivalent formulation is

$$
\exists L>0,0<\mu<1: \quad\left\|\left(\mathrm{S}^{[1]}\right)^{L} f-\left(S^{[1]}\right)^{L} g\right\|_{\infty}=\mu\|f-g\|_{\infty}, \quad \forall f, g \in l_{\infty}(\mathbb{Z}) .
$$

Then, according to (34), the key to stability lies in the ability to bound products of Generalized Jacobians of the first difference scheme $S^{[1]}$. Let us denote

$$
A:=A(w)=D S^{[1]}(w), \quad w \in l_{\infty}(\mathbb{Z}) .
$$

Following (32) and (26)-(27), we get that the generalized Jacobian of $S^{[1]}$ has the following non-zero entries, $\forall n \in \mathbb{Z}$,

$$
\begin{aligned}
\left(D \mathrm{~S}^{[1]}(w)\right)_{[2 n, n-1: n+1]} & =D \psi_{0}\left(w_{n-1}, w_{n}, w_{n+1}\right) \\
\left(D \mathrm{~S}^{[1]}(w)\right)_{[2 n+1, n-1: n+1]} & =D \psi_{1}\left(w_{n-1}, w_{n}, w_{n+1}\right)
\end{aligned}
$$

Taking into account (17)-(13), we have that these entries are either zero or depend on the parameters

$$
r:=\frac{w_{n}-w_{n-1}}{w_{n}+w_{n-1}}, \quad \tilde{r}:=\frac{w_{n+1}-w_{n}}{w_{n+1}+w_{n}} .
$$

Considering the 'worst' possible case (all non-zero components are non-zero) we can write

$$
\begin{aligned}
8\left(\begin{array}{lll}
A_{2 n, n-1} & A_{2 n, n} & A_{2 n, n+1}
\end{array}\right) & =\left(\begin{array}{lll}
\phi(r) & 4+\phi(-r)-\phi(\tilde{r}) & -\phi(-\tilde{r})
\end{array}\right) \\
8\left(\begin{array}{llll}
A_{2 n+1, n-1} & A_{2 n+1, n} & A_{2 n+1, n+1}
\end{array}\right) & =\left(\begin{array}{lll}
-\phi(r) & 4-\phi(-r)+\phi(\tilde{r}) & \phi(-\tilde{r})
\end{array}\right)
\end{aligned}
$$

It is easy to see that $A_{2 n, n-1} \geq 0, A_{2 n, n+1} \leq 0, A_{2 n+1, n-1} \leq 0, A_{2 n+1, n+1} \geq 0$,

$$
1 / 4 \leq A_{2 n, n} \leq 3 / 4, \quad 1 / 4 \leq A_{2 n+1, n} \leq 3 / 4
$$

$(0 \leq \phi( \pm t) \leq 2, \forall t \in[-1,1]$, see Figure 1$)$. Thus, the components of $A_{[2 n,:]}, A_{[2 n+1,:]}$ do not change sign, and we can write

$$
\begin{aligned}
\left\|A_{[2 n,:]}\right\|_{1} & =\frac{1}{8}(\phi(r)+4+(\phi(-r)-\phi(\tilde{r}))+\phi(-\tilde{r}))=\frac{1}{8}(4+\psi(r)-\rho(\tilde{r})) \\
\left\|A_{[2 n+1,:]}\right\|_{1} & =\frac{1}{8} \phi(r)+\frac{1}{2}-\frac{1}{8}(\phi(-r)-\phi(\tilde{r}))+\frac{1}{8} \phi(-\tilde{r})=\frac{1}{2}+\frac{1}{8}(\rho(r)+\psi(\tilde{r}))
\end{aligned}
$$

where

$$
\psi(t):=\phi(t)+\phi(-t)=(1+t)^{2}, \quad \rho(t):=\phi(t)-\phi(-t)=2 t .
$$

The functions involved are plotted on $[-1,1]$ in Figure 1 . Using the appropriate bounds, we easily get

$$
\left\{\begin{array}{ll}
\left\|A_{[2 n,:]}\right\|_{1} & \leq 1, \\
\left\|A_{[2 n+1,:]}\right\|_{1} & \leq 1
\end{array} \rightarrow \quad\left\|D S^{[1]}(w)\right\|_{\infty}=\sup _{n \in \mathbb{Z}}\left\{\left\|A_{2 n}\right\|_{1},\left\|A_{2 n+1}\right\|_{1}\right\} \leq 1\right.
$$

No smaller bound can be found by this technique, since the bounds above could be attained (for $r=$ $1, \tilde{r}=-1)$.

Let us consider next the product of two generalized gradients,

$$
\left\|D S^{[1]}(w) D S^{[1]}(u)\right\|_{\infty}=\sup _{n \in \mathbb{Z}} \max \left\{\left\|B_{[4 n+k,:]}\right\|_{1}, k=0,1,2,3\right\}, \quad w, u \in l_{\infty}(\mathbb{Z}) .
$$

where, as before, we use the following notation

$$
B=A \tilde{A}, \quad A:=D S^{[1]}(w), \quad \tilde{A}:=D S^{[1]}(u) .
$$



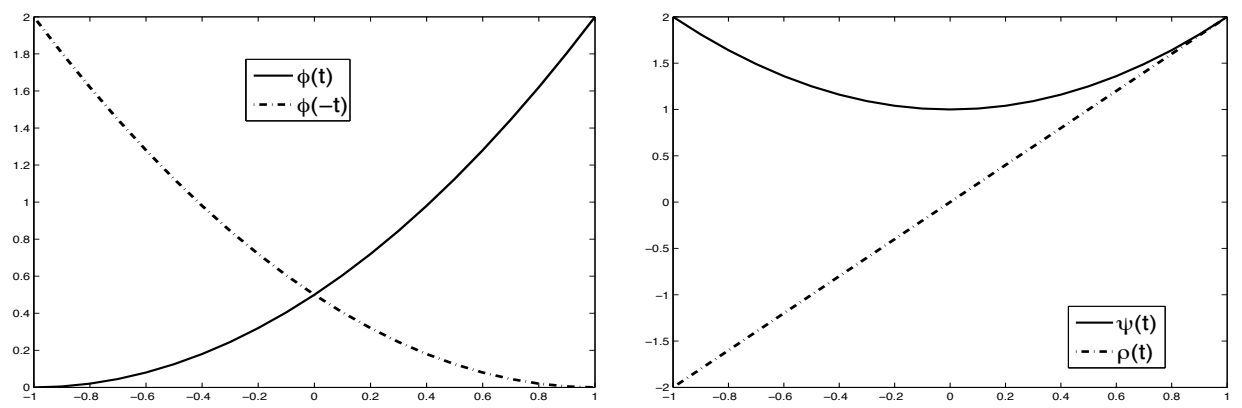

Figure 1: Graphs of $\phi(t)=(1+t 2) / 2, \phi(-t), \rho(t)=\phi(t)-\phi(-t)=2 t$ and $\psi(t)=\phi(t)+\phi(-t)=(1+t)^{2}$

To compute the non-zero entries of $B_{[4 n+k,:]}$ for $k=0,1$ we carry out the matrix product

$$
\left(\begin{array}{ccc}
A_{4 n, 2 n-1} & A_{4 n, 2 n} & A_{4 n, 2 n+1} \\
A_{4 n+1,2 n-1} & A_{4 n+1,2 n} & A_{4 n+1,2 n+1}
\end{array}\right)\left(\begin{array}{cccc}
\tilde{A}_{2 n-1, n-2} & \tilde{A}_{2 n-1, n-1} & \tilde{A}_{2 n-1, n} & 0 \\
0 & \tilde{A}_{2 n, n-1} & \tilde{A}_{2 n, n} & \tilde{A}_{2 n, n+1} \\
0 & \tilde{A}_{2 n+1, n-1} & \tilde{A}_{2 n+1, n} & \tilde{A}_{2 n+1, n+1}
\end{array}\right)
$$

while the corresponding non-zero entries for $k=2,3$, are computed from

$$
\left(\begin{array}{ccc}
A_{4 n+2,2 n} & A_{4 n+2,2 n+1} & A_{4 n+2,2 n+2} \\
A_{4 n+3,2 n} & A_{4 n+3,2 n+1} & A_{4 n+3,2 n+2}
\end{array}\right)\left(\begin{array}{cccc}
\tilde{A}_{2 n, n-1} & \tilde{A}_{2 n, n} & \tilde{A}_{2 n, n+1} & 0 \\
\tilde{A}_{2 n+1, n-1} & \tilde{A}_{2 n+1, n} & \tilde{A}_{2 n+1, n+1} & 0 \\
0 & \tilde{A}_{2 n+2, n} & \tilde{A}_{2 n+2, n+1} & \tilde{A}_{2 n+2, n+2}
\end{array}\right)
$$

As before, the entries in the matrices above are either zero or can be written in terms of the following parameters

$$
\begin{gathered}
s:=\frac{w_{2 n}-w_{2 n-1}}{w_{2 n}+w_{2 n-1}} \quad \tilde{s}:=\frac{w_{2 n+1}-w_{2 n}}{w_{2 n+1}+w_{2 n}} \quad \bar{s}:=\frac{w_{2 n+2}-w_{2 n+1}}{w_{2 n+2}+w_{2 n+1}} \\
\hat{t}:=\frac{u_{n-1}-u_{n-2}}{u_{n-1}+u_{n-2}} \quad t:=\frac{u_{n}-u_{n-1}}{u_{n}+u_{n-1}} \quad \tilde{t}:=\frac{u_{n+1}-u_{n}}{u_{n+1}+u_{n}} \quad \bar{t}:=\frac{u_{n+2}-u_{n+1}}{u_{n+2}+u_{n+1}}
\end{gathered}
$$

It is straightforward (but cumbersome) to bound the components of $B_{4 n+k}, k=0,1,2,3$. We illustrate the procedure by considering next the cases $k=0,1$ (the remaining cases are similar (see [27]). For this, we consider again the worst possible case, and observe that (39) gives

$$
\begin{aligned}
& \left(\begin{array}{c}
B_{[4 n, n-2: n+1]} \\
B_{[4 n+1, n-2: n+1]}
\end{array}\right)=\frac{1}{64} C \cdot D
\end{aligned}
$$

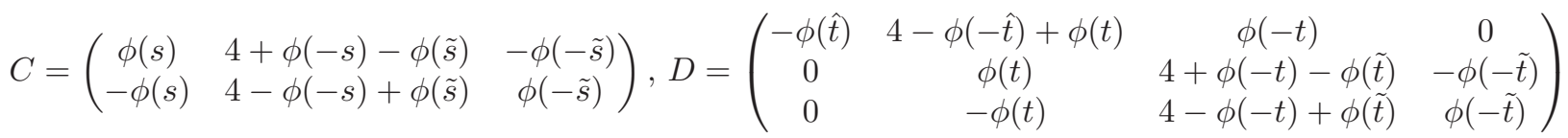

Case $\mathbf{k}=\mathbf{0}$. We can write $64 B_{[4 n, n-2: n+1]}=\left(P_{1}, P_{2}, P_{3}, P_{4}\right)$ with

$$
\begin{aligned}
& P_{1}=-\phi(s) \phi(\hat{t}) \leq 0 \\
& \left.P_{2}=\phi(t)(4+\psi(s)-\rho(\tilde{s}))+\phi(s)(4-\phi(-\hat{t}))\right) \geq 0 \\
& P_{3}=\phi(-t)(4+\psi(s)-\rho(\tilde{s}))+4(4+\phi(-s)-\psi(\tilde{s}))-\phi(\tilde{t})(4+\phi(-s)-\rho(\tilde{s})) \\
& P_{4}=-\phi(-\tilde{t})(4+\phi(-s)-\rho(\tilde{s})) \leq 0 .
\end{aligned}
$$


Using the bounds on $\phi, \psi, \rho$ from Figure 1 , we easily get that $P_{1} \leq 0, P_{2} \geq 0, P_{4} \leq 0$, but $P_{3}$ does not have a definite sign, hence

$$
\left\|B_{[4 n,:]}\right\|_{1}=\frac{1}{64} S_{4 n}, \quad S_{4 n}:=\left(-P_{1}+P_{2}+\left|P_{3}\right|-P_{4}\right)
$$

- $\left|P_{3}\right|=P_{3} \rightarrow S_{4 n}:=-P_{1}+P_{2}+P_{3}-P_{4}$. We write

$$
\begin{aligned}
& -P_{1}+P_{2}=\phi(t)(4+\psi(s)-\rho(\tilde{s}))+\phi(s)(4+\rho(\hat{t})) \\
& \quad P_{3}-P_{4}=\phi(-t)(4+\psi(s)-\rho(\tilde{s}))+4(4+\phi(-s)-\psi(-\tilde{s}))-\rho(\tilde{t})(4+\phi(-s)-\rho(\tilde{s})) \\
& S_{4 n}=\psi(t)(4+\psi(s)-\rho(\tilde{s}))+\phi(s) \rho(\hat{t})+4(4+\psi(s)-\psi(\tilde{s}))-\rho(\tilde{t})(4+\phi(-s)-\rho(\tilde{s}))
\end{aligned}
$$

Taking into account the bound for $\phi, \psi, \rho$, we easily deduce

$$
3 \leq 4+\psi(s)-\rho(\tilde{s}) \leq 8, \quad 3 \leq 4+\psi(s)-\psi(\tilde{s}) \leq 4, \quad 0 \leq 4+\phi(-s)-\rho(\tilde{s}) \leq 8
$$

hence

$$
S_{4 n} \leq 2 \cdot 8+2 \cdot 2+4 \cdot 4+2 \cdot 8=52 \quad \Rightarrow \quad\left\|B_{[4 n,:]}\right\|_{1}=\frac{52}{64}<1
$$

- $\left|P_{3}\right|=-P_{3} \rightarrow S_{4 n}:=-P_{1}+P_{2}-P_{3}-P_{4}$. Now, we write

$$
-P_{3}-P_{4}=-\phi(-t)(4+\psi(s)-\rho(\tilde{s}))-4(4+\phi(-s)-\psi(-\tilde{s}))+\psi(\tilde{t})(4+\phi(-s)-\rho(\tilde{s}))
$$

Then,

$$
S_{4 n}=\rho(t)(4+\psi(s)-\rho(\tilde{s}))+\phi(s) \rho(\hat{t})+\psi(\tilde{t})(4+\phi(-s)-\rho(\tilde{s}))-4(4-\rho(s)-\psi(\tilde{s})) .
$$

Proceeding as before, and taking into account that $4-\rho(s)-\psi(\tilde{s} \geq 0$,

$$
S_{4 n} \leq 2 \cdot 8+2 \cdot 2+0+2 \cdot 8=36 \quad \Rightarrow \quad\left\|B_{[4 n,:]}\right\|_{1} \leq \frac{52}{64} \leq 1
$$

Case $\mathbf{k}=1$. Now $64 B_{[4 n+1, n-2: n+1]}=\left(P_{1}, P_{2}, P_{3}, P_{4}\right)$ with

$$
\begin{aligned}
& P_{1}=\phi(s) \phi(\hat{t}) \geq 0 \\
& P_{2}=\phi(t)(4-\psi(s)+\rho(\tilde{s}))-\phi(s)(4-\phi(-\hat{t})) \\
& P_{3}=\phi(-t)(4-\psi(s)+\rho(\tilde{s}))+(4-\phi(-s)+\phi(\tilde{s}))(4-\phi(\tilde{t}))+\phi(-\tilde{s})(4+\phi(\tilde{t})) \geq 0 \\
& P_{4}=-\phi(-\tilde{t})(4-\phi(-s)+\rho(\tilde{s})) \leq 0
\end{aligned}
$$

In this case, $P_{2}$ may have either sign, hence

$$
\left\|B_{[4 n+1,:]}\right\|_{1}=\frac{1}{64} S_{4 n+1}, \quad S_{4 n+1}:=\left(P_{1}+\left|P_{2}\right|+P_{3}-P_{4}\right)
$$

- $\left|P_{2}\right|=P_{2} \rightarrow S_{4 n+1}=P_{1}+P_{2}+P_{3}-P_{4}$.

$$
\begin{gathered}
P_{1}+P_{2}=\phi(t)(4-\psi(s)+\rho(\tilde{s}))-\phi(s)(4-\psi(\hat{t})) \\
P_{3}-P_{4}=\phi(-t)(4-\psi(s)+\rho(\tilde{s}))+4(4-\phi(-s)+\psi(\tilde{s}))-\rho(\tilde{t})(4-\phi(-s)+\rho(\tilde{s})) \\
S_{4 n+1}=-\phi(s)(4-\psi(\hat{t}))+\psi(t)(4-\psi(s)+\rho(\tilde{s}))+4(4-\phi(-s)+\psi(\tilde{s}))-\rho(\tilde{t})(4-\phi(-s)+\rho(\tilde{s})) .
\end{gathered}
$$

The bound for $\phi, \psi, \rho$ (Figure 1) lead to

$$
0 \leq 4-\psi(s)+\rho(\tilde{s}) \leq 5, \quad 3 \leq 4-\phi(-s)+\psi(\tilde{s}) \leq 6, \quad 0 \leq 4-\phi(-s)+\rho(\tilde{s}) \leq 6,
$$




$$
S_{4 n+1} \leq 0+2 \cdot 5+4 \cdot 6+2 \cdot 6=46 \quad \rightarrow \quad\left\|B_{[4 n+1,:]}\right\|_{1} \leq \frac{46}{64}<1 .
$$

- $\left|P_{2}\right|=-P_{2} \rightarrow S_{4 n+1}=P_{1}-P_{2}+P_{3}-P_{4}$. Now, we can write

$$
\begin{gathered}
P_{1}-P_{2}=\phi(s)(4+\rho(\hat{t}))-\phi(t)(4-\psi(s)+\rho(\tilde{s})) \\
S_{4 n+1}=\phi(s)(4+\rho(\hat{t}))-\rho(t)(4-\psi(s)+\rho(\tilde{s}))+4(4-\phi(-s)+\psi(\tilde{s}))-\rho(\tilde{t})(4-\phi(-s)+\rho(\tilde{s})) \\
S_{4 n+1} \leq 2 \cdot 6+2 \cdot 5+4 \cdot 6+2 \cdot 6=58 \quad \Rightarrow \quad\left\|B_{[4 n+1,:]}\right\|_{1} \leq \frac{58}{64}<1
\end{gathered}
$$

Proceeding in an analogous way, we can prove that $\left\|B_{4 n+3}\right\|_{\infty} \leq 56 / 64,\left\|B_{4 n+2}\right\|_{\infty} \leq 58 / 64$, Hence

$$
\left\|D \mathrm{~S}^{[1]}(w) D \mathrm{~S}^{[1]}(u)\right\|_{1} \leq \frac{58}{64}<1, \quad \forall u, w \in l_{\infty}(\mathbb{Z}) .
$$

and, according to (34), we have that

$$
\left\|\left(S^{[1]}\right)^{2} f-\left(S^{[1]}\right)^{2} g\right\|_{\infty} \leq \frac{58}{64}\|f-g\|_{\infty}
$$

so that condition $\mathbf{S} 2$ is satisfied with $L=2$.

\subsection{Order of Approximation of the PCHIP subdivision scheme}

The stability of the PCHIP subdivision scheme allows us to examine in a straightforward manner the approximation properties of the scheme.

Theorem 8. Let $f=\left\{f_{i}\right\}_{i \in \mathbb{Z}}, f_{i}=f\left(x_{i}\right), x_{i+1}-x_{i}=h, f$ smooth. Then

1. $\left\|S^{\infty} f-f\right\|_{\infty}=O\left(h^{2}\right)$.

2. If $f$ is strictly monotone, then $\left\|S^{\infty} f-f\right\|_{\infty}=O\left(h^{4}\right)$.

Proof. The PCHIP scheme is stable and reproduces exactly polynomials of degree 1 , hence item 1 follows from Theorem 2.

In order to prove item 2 , we show first that if $f$ is strictly monotone, then

$$
\left\|\mathrm{S} f-S_{2,2} f\right\|_{\infty}=O\left(h^{4}\right),
$$

where $S_{2,2}$ is the 4-point interpolatory scheme of Deslauries-Dubuc. Since the order of approximation after one iteration of $S_{2,2}$ is equal to 4 , we have

$$
\|\mathrm{S} f-f\|_{\infty} \leq\left\|\mathrm{S} f-S_{2,2} f\right\|_{\infty}+\left\|S_{2,2} f-f\right\|_{\infty}=O\left(h^{4}\right)
$$

hence the result follows, again, from Theorem 2.

In order to prove (41), we notice first that for $x, y, z$ of the same sign

$$
\mathrm{H}(x, y)-\mathrm{H}(y, z)=2\left(\frac{x y}{x+y}-\frac{y z}{y+z}\right)=2 \frac{(x-z) y^{2}}{(x+y)(y+z)},
$$

and also that $S_{2,2}$ can be equivalently written as follows

$$
\left(S_{2,2} f\right)_{2 n+1}=\frac{1}{2}\left(f_{n+1}+f_{n}\right)-\frac{1}{8}\left(\frac{\nabla^{2} f_{n-1}+\nabla^{2} f_{n}}{2}\right)
$$

Since $\nabla^{2} f_{n-1}+\nabla^{2} f_{n}=\nabla f_{n+1}-\nabla f_{n-1}$, using (42) we may write

$$
(\mathrm{S} f)_{2 n+1}-\left(S_{2,2} f\right)_{2 n+1}=\frac{1}{16}\left(\nabla f_{n+1}-\nabla f_{n-1}\right)\left(1-\frac{4\left(\nabla f_{n}\right)^{2}}{\left(\nabla f_{n-1}+\nabla f_{n}\right)\left(\nabla f_{n}+\nabla f_{n+1}\right)}\right)
$$

The result follows from straightforward Taylor expansions, which show that for smooth data

$$
\begin{aligned}
\nabla f_{n+1}-\nabla f_{n-1} & =O\left(h^{2}\right), \\
\left(\nabla f_{n-1}+\nabla f_{n}\right)\left(\nabla f_{n}+\nabla f_{n+1}\right)-4\left(\nabla f_{n}\right)^{2} & =O\left(h^{4}\right)
\end{aligned}
$$

while for strictly monotone data $\left(\nabla f_{n-1}+\nabla f_{n}\right)\left(\nabla f_{n}+\nabla f_{n+1}\right)=O\left(h^{2}\right)$. 


\begin{tabular}{|c|c|c|c|c|}
\hline $\mathrm{h}$ & $\left\|S_{1,1} f-f\right\|_{\infty}$ & $\left\|S_{2,2} f-f\right\|_{\infty}$ & $\|\mathrm{S} f-f\|_{\infty}$ & $\left\|S_{\mathrm{H}_{2}} f-f\right\|_{\infty}$ \\
\hline 0.1 & $1.8 e-3$ & $5.4 e-5$ & $5.3 e-7$ & $4.8 e-4$ \\
0.05 & $4.6 e-4$ & $3.4 e-6$ & $4.0 e-8$ & $6.1 e-5$ \\
0.025 & $1.2 e-4$ & $2.1 e-7$ & $2.7 e-9$ & $7.7 e-6$ \\
0.0125 & $3.2 e-5$ & $1.3 e-8$ & $1.7 e-10$ & $9.6 e-7$ \\
\hline$q$ & 1.9 & 4.0 & 3.8 & 2.9 \\
\hline
\end{tabular}

Table 1: Approximation order study on the interval $[-1,-0.3]$ for gaussian data. $\left\|S^{\infty} f-f\right\|_{\infty} \approx C h^{q}$.

\section{Numerical experiments}

In this section we perform a series of numerical experiments that confirm and illustrate the theoretical results about the PCHIP subdivision scheme proven in the previous sections. For the sake of comparison, we shall consider other schemes in the same framework, i.e. interpolatory schemes that can be expressed as specified in (2). In particular, we consider the linear two-point scheme $S_{1,1}$, which is monotone but only first order accurate, the four-point DD scheme $S_{2,2}$ and the PPH scheme, (Power 2$) S_{\mathrm{H}_{2}}$, described in [2]. We recall that the PPH scheme, the 4-point DD scheme, and the PCHIP scheme proposed in this paper are all based on cubic piecewise polynomial interpolatory techniques and have the same 4-point stencil.

\subsection{Approximation order}

We consider a Gaussian function $f(x)$ with $\mu=0 \sigma=0.5$, which we sample on an initial grid with a fixed step-size $h$. We measure $\left\|S^{\infty} f^{0}-f^{0}\right\|_{\infty}$ (taking $S^{\infty} f^{0}$ as $S^{L} f^{0}$ with $L=7$ ) and compute the order of accuracy by a (log-log) least square fit, assuming $\left\|S^{\infty} f^{0}-f^{0}\right\|_{\infty}=O\left(h^{q}\right)$.

In Table 1, we display the errors, and the orders of approximation, on the interval $[-1,-0.3]$, which correspond to monotone (and slowly varying) data, and in Table 2 we display the results and approximation orders corresponding to the interval the interval $[-0.4,0.4]$, a convex, non-monotone, region. In both tables we observe the expected order of approximation of the schemes considered (the PPH scheme is fourth order accurate for smooth convex data [14]). The computed order of accuracy of the PCHIP scheme in the case of monotone data is 4, while only a second order approximation is obtained for non-monotone data, as expected from Theorem 8.

\begin{tabular}{|c|c|c|c|c|}
\hline $\mathrm{h}$ & $\left\|S_{1,1} f-f\right\|_{\infty}$ & $\left\|S_{2,2} f-f\right\|_{\infty}$ & $\|\mathrm{S} f-f\|_{\infty}$ & $\left\|S_{\mathrm{H}_{2}} f-f\right\|_{\infty}$ \\
\hline 0.1 & $5.4 e-5$ & $3.4 e-6$ & $9.8 e-4$ & $1.9 e-4$ \\
0.05 & $1.5 e-5$ & $2.4 e-7$ & $2.4 e-4$ & $1.4 e-5$ \\
0.025 & $4.2 e-6$ & $1.5 e-8$ & $6.2 e-5$ & $9.7 e-7$ \\
0.0125 & $1.1 e-6$ & $1.0 e-9$ & $1.5 e-5$ & $6.4 e-8$ \\
\hline$q$ & 1.8 & 3.9 & 1.9 & 3.8 \\
\hline
\end{tabular}

Table 2: Approximation order study the interval $[-0,4,0,4]$ for gaussian data. $\left\|S^{\infty} f-f\right\|_{\infty} \approx C h^{q}$.

\subsection{Shape preservation}

The next example shows that monotonicity preservation avoids undesirable oscillatory behavior. We consider the initial data given in Table 3, and display the limit functions $S^{\infty} f$ for different subdivision schemes in Figure 2.

As expected, the $S_{2,2}$ scheme generates visible oscillations in the form of over and undershoots. The PPH scheme, $S_{\mathrm{H}_{2}}$, is specifically designed to avoid oscillatory behavior, however the lack of smoothness of the limit function for the data of Table 3 is evident. This seems to be always the case when refining monotone data with large gradients and convexity changes with this scheme. 


\begin{tabular}{|c|c|c|c|c|c|c|c|c|c|c|c|c|c|c|c|c|c|}
\hline$x$ & 1 & 2 & 3 & 4 & 5 & 6 & 7 & 8 & 9 & 10 & 11 & 12 & 13 & 14 & 15 & 16 & 17 \\
\hline$f(x)$ & 10 & 10 & 10 & 10 & 10 & 10.5 & 10.5 & 10.5 & 10.5 & 15 & 50 & 50 & 50 & 50 & 60 & 85 & 85 \\
\hline
\end{tabular}

Table 3: Staircase data.
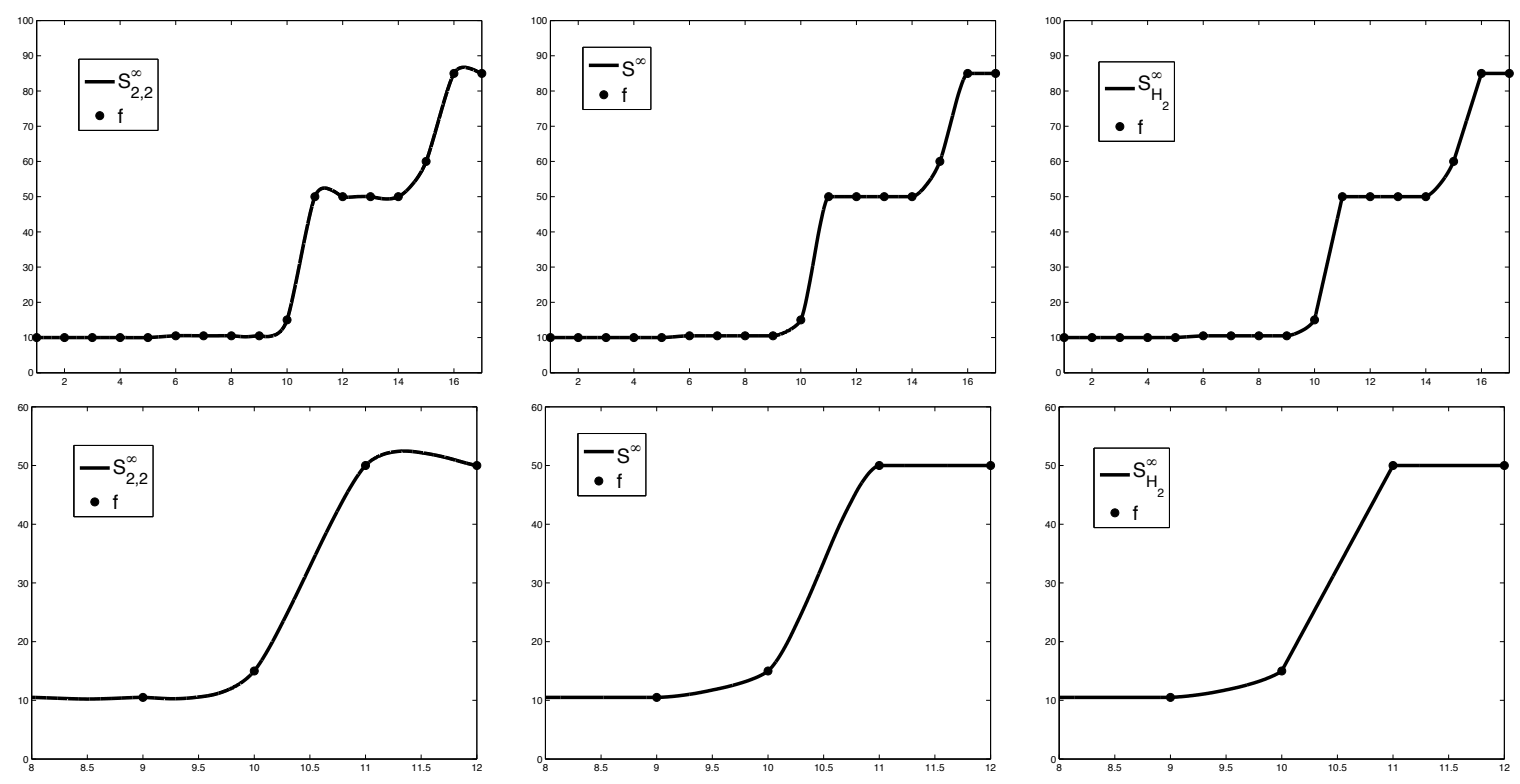

Figure 2: (•) Staircase data and limit functions. Initial samples are marked as black dots.

As stated in previous sections, the PCHIP subdivision scheme is derived from an interpolatory reconstruction that preserves the monotonicity of the data, hence it was expected to be monotonicity preserving, a fact which was proven in Lemma 4 by considering the first difference scheme and confirmed by the results shown in Figure 2.

After completing the derivations in this paper we became aware that the PCHIP subdivision process corresponds to one particular choice of parameters in the family of (piecewise) monotonicity preserving interpolatory subdivision schemes described in [24]. In the notation of this paper, this scheme can be described as follows:

$$
\left(S_{K} f^{k+1}\right)_{2 n+1}=\frac{f_{n}^{k}+f_{n+1}^{k}}{2}+\frac{1}{2} \nabla f_{n}^{k} G\left(r_{n}^{k}, R_{n+1}^{k}\right), \quad r_{n}^{k}=\frac{\nabla f_{n-1}^{k}}{\nabla f_{n}^{k}}, \quad R_{n}^{k}=\frac{\nabla f_{n}^{k}}{\nabla f_{n-1}^{k}}
$$

with

$$
G(r, R)=\frac{r-R}{l_{1}+\left(1+l_{2}\right)(r+R)+l_{3} R}, \quad l_{1}=2, l_{2}=1, l_{3}=2
$$

We notice that for $x, y, z$ non zero and of the same sign it is straightforward to show that (see (42))

$$
\frac{1}{2} y G\left(\frac{x}{y}, \frac{z}{y}\right)=\frac{1}{4} \frac{(x-z) y^{2}}{(x+y)(y+z)}=\frac{1}{8}(H(x, y)-H(y, z)),
$$

hence both schemes are equivalent for monotone data.

It is shown in [24] that this scheme produces $C^{1}$ functions when applied to monotone data, which explains the smooth behavior observed in Figure 3. For general (non-monotone) data, it is also easy to show that the two schemes coincide provided the following function is used in (43),

$$
G_{P M}(r, R):=G(\max 0, r, \max 0, R)
$$


We close this section by considering the case of under-resolved, convex data. The initial data shown in Figure 3) correspond to the same Gaussian function as in section 5.1, sampled on an equally spaced grid with $h=1$. The PPH scheme, which is convexity preserving and fourth order accurate on smooth convex data, produces a smooth limit function, as shown in 3. However, the 'monotonicity preservation' property seems to lead to the clipping of non-resolved maxima.
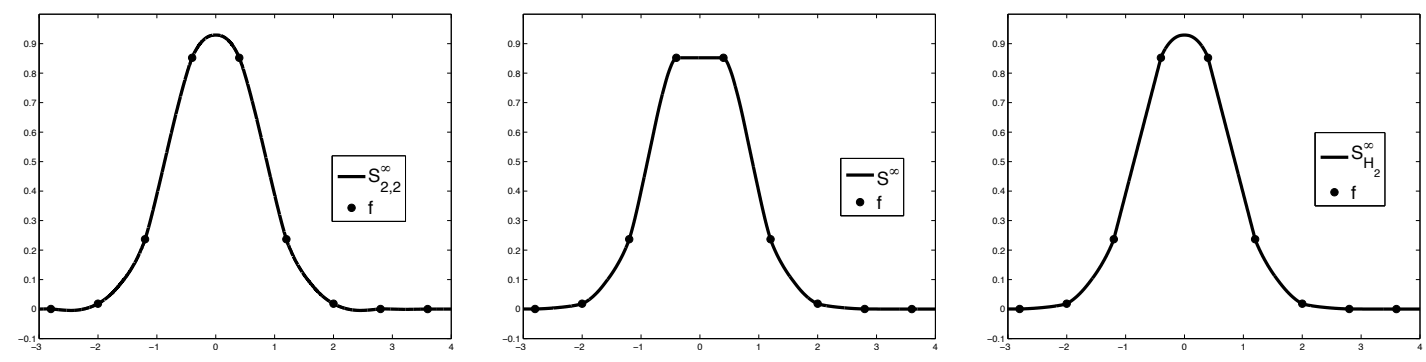

Figure 3: (Refinement of coarse Gaussian Data. The initial samples are marked as black dots in the figure.

\section{Conclusion}

We have constructed a monotonicity preserving subdivision scheme using the monotonicity preserving piecewise polynomial Hermite-type interpolatory technique implemented in the PCHIP package in Matlab a basic building block. The interpolatory technique is third order accurate for smooth monotone data, but fourth order accurate at the midpoint of the considered interval, which allowed us to prove that the PCHIP subdivision scheme is fourth order accurate on monotone data. We have studied its convergence and stability by exploiting the (piecewise) smoothness properties of the functions that define the subdivision scheme, following a novel technique developed in [20,27], which is based on obtaining appropriate bounds for the generalized Jacobian of the first difference scheme and products of the associated linear maps.

We have a performed several numerical experiments that confirm the theoretical results stated in this paper. In the revision process, we realized that the PCHIP subdivision scheme is equivalent to one member of a family of monotonicity preserving subdivision schemes studied in [24]. We remark, however that the design and analysis carried out in this paper is essentially different from that in [24], emphasizing the relationship between a monotone interpolatory technique and its associated subdivision scheme, in the design part, and making use of a very interesting and novel tool based on an extended differential calculus for a certain class of piecewise smooth functions in the analysis part.

Acknowledgments. The authors acknowledge support from MTM2011-22741.

\section{References}

[1] S. Amat, K. Dadourian, and J. Liandrat. Analysis of a class of nonlinear subdivision schemes and associated multiresolution transforms. Adv. Comput. Math., 34(3):253-277, 2011.

[2] S. Amat, R. Donat, J. Liandrat, and J.C. Trillo. Analysis of a new nonlinear subdivision scheme. applications in image processing. Applications in Image Processing. Found. Comput. Math., pages $193-225,2006$.

[3] S. Amat, R. Donat, J. Liandrat, and J.C. Trillo. A fully adaptive multiresolution scheme for image processing. Math. Comput. Modelling, 46:2-11, 2007.

[4] S. Amat and J. Liandrat. On the stability of the pph nonlinear multiresolution. Appl. Comput. Harmon. Anal., 18(2):198-206, 2005. 
[5] F. Aràndiga, R. Donat, and A. Harten. Multiresolution based on weighted averages of the hat function I: linear reconstruction tecniques. SIAM J. Numer. Anal., 36:160-203, 1999.

[6] F. Aràndiga. On the order of nonuniform monotone cubic hermite interpolants. SIAM J. Numer. Anal., 51,5:2613-2633, 2013.

[7] F. Aràndiga, A. M. Belda, and P. Mulet. Point-value WENO multiresolution applications to stable image compression. J Sci Comput, 43:158-182, 2010.

[8] F. Aràndiga and R. Donat. Nonlinear multi-scale decomposition: the approach of A. Harten. Numer. Algorithms, 23:175-216, 2000.

[9] F. Aràndiga and R. Donat. Stability through synchronization in nonlinear multiscale transformations. SIAM J. Sci. Comput., 29(1):265-289, 2007.

[10] C. De Boor and B. Swartz. Piecewise monotone interpolation. J. Approx. Theory, 21:411-416, 1977.

[11] Richard L. Burden and J. Douglas Faires. Numerical Analysis. Thomson Brooks/Cole, 2005.

[12] A. S. Cavaretta, W. Dahmen, and C.A. Michelli. Stationary subdivision. Mem. Amer. Math. Soc. 93, 93(453):346-349, 1991.

[13] A. Cohen, N. Dyn, and B. Matei. Quasilinear subdivision schemes with applications to ENO interpolation. Appl. Comput. Harmon. Anal, 15:89-116, 2003.

[14] K. Dadourian. Schémas de Subdivision, Analyses Multirésolutions non-linéaires. Applications. PhD thesis, Université de Provence, 2008.

[15] K. Dadourian and J. Liandrat. Analysis of some bivariate non-linear interpolatory subdivision schemes. Numer. Algorithms, 48:261-278, 2008.

[16] G. Deslauriers and S. Dubuc. Interpolation dyadique: Fractals, dimension non entieres et application. Masson, Paris, pages 44-45, 1987.

[17] N. Dyn. Subdivision schemes in computer-aided geometric design. Advances in numerical analysis, Vol. II (Lancaster, 1990), Oxford Sci. Publ., Oxford Univ. Press, New York, pages 36-104, 1992.

[18] N. Dyn and P. Oswald. Univariante subdivision and multi-scale transforms: the nonlinear case. R.A. DeVore, A. Kunoth (eds.), Multiscale, Nonlinear and Adaptive Approximation (C) Springer-Verlag Berlin Heidelberg, pages 203-245, 2009.

[19] F. N. Fritsch and R. E. Carlson. Piecewise cubic interpolation. SIAM J. Num. Anal., 17, 2:238-246, 1980.

[20] S. Harizanov and P.Oswald. Stability of nonlinear subdivision and multiscale transforms. Constr. Approx., 31(3):359-393, 2010.

[21] A. Harten. Multiresolution representation of data: a general framework. SIAM J. Numer. Anal., $33: 1205-1256,1996$.

[22] H. T. Huynh. Accurate monotone cubic interpolation. SIAM J. Numer. Anal., 30:57-100, 1993.

[23] F. Kuijt. Convexity preserving interpolation. Stationary nonlinear subdivision and splines. PhD thesis, University of Twente (The Netherlands), 1998.

[24] F. Kuijt and R. Van Damme. Monotonicity preserving interpolatory subdivision scheme. J. Comput. Appl. Math., 101:203-229, 1999. 
[25] A. Marquina and S. Serna. Power ENO methods: a fifth-order accurate weighted power ENO method. J. Comput. Phys., 194(2):632-658, 2004.

[26] C. Moler. Numerical computing with MATLAB. SIAM, Philadelphia, 2004.

[27] M. Santágueda. Metodos multiescala y aplicaciones. PhD thesis, Universitat de València, 2014.

[28] Gang Xie and Thomas P.-Y. Yu. Smoothness analysis of nonlinear subdivision schemes of homogeneous and affine invariant type. Constr. Approx., 22:219-254, 2005. 